\title{
BMJ Open First results about recovery of walking function in patients with intensive care unit-acquired muscle weakness from the General Weakness Syndrome Therapy (GymNAST) cohort study
}

\author{
Jan Mehrholz, ${ }^{1,2}$ Simone Mückel, ${ }^{1}$ Frank Oehmichen, ${ }^{3}$ Marcus Pohl ${ }^{3}$
}

To cite: Mehrholz J,

Mückel S, Oehmichen F, et al. First results about recovery of walking function in patients with intensive care unitacquired muscle weakness from the General Weakness Syndrome Therapy (GymNAST) cohort study. BMJ Open 2015;5:e008828. doi:10.1136/bmjopen-2015008828

- Prepublication history for this paper is available online. To view these files please visit the journal online (http://dx.doi.org/10.1136/ bmjopen-2015-008828).

Received 19 May 2015 Revised 29 October 2015 Accepted 3 November 2015

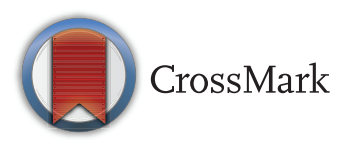

${ }^{1}$ Wissenschaftliches Institut, Private Europäische Medizinische Akademie der Klinik Bavaria in Kreischa, Kreischa, Germany ${ }^{2}$ Department of Public Health, Medizinische Fakultät 'Carl Gustav Carus', Technische Universität Dresden, Dresden, Germany

${ }^{3}$ Fach und Privatkrankenhaus, Klinik Bavaria in Kreischa, Kreischa, Germany

\section{Correspondence to} Professor Jan Mehrholz; jan.mehrholz@klinik-bavaria. de

\section{ABSTRACT}

Objectives: To describe the time course of recovery of walking function and other activities of daily living in patients with intensive care unit (ICU)-acquired muscle weakness.

Design: This is a cohort study.

Participants: We included critically ill patients with ICU-acquired muscle weakness.

Setting: Post-acute ICU and rehabilitation units in Germany.

Measures: We measured walking function, muscle strength, activities in daily living, motor and cognitive function.

Results: We recruited 150 patients ( $30 \%$ female) who fulfilled our inclusion and exclusion criteria. The primary outcome recovery of walking function was achieved after a median of 28.5 days $(I Q R=45)$ after rehabilitation onset and after a median of 81.5 days $(I Q R=64)$ after onset of illness. Our final multivariate model for recovery of walking function included two clinical variables from baseline: the Functional Status Score ICU (adjusted HR=1.07 (95\% Cl 1.03 to 1.12) and the ability to reach forward in $\mathrm{cm}$ (adjusted $\mathrm{HR}=1.02(95 \% \mathrm{Cl} 1.00$ to 1.04$)$. All secondary outcomes but not pain improved significantly in the first 8 weeks after study onset.

Conclusions: We found good recovery of walking function for most patients and described the recovery of walking function of people with ICU-acquired muscle weakness.

Trials registrations number: Sächsische Landesärztekammer EK-BR-32/13-1; DRKS00007181, German Register of Clinical Trials.

\section{INTRODUCTION}

In clinical practice, it is often seen that critically ill patients in an intensive care unit (ICU) get weak muscles. According to Nordon-Craft, this weakness is characterised by a profound weakness that is greater than might be expected to result from prolonged bed rest ${ }^{1}$ and therefore designates clinically detected weakness in critically ill patients in

\section{Strengths and limitations of this study}

- Strengths include that a precise daily documentation of the recovery of walking function in the first months of intensive care unit (ICU)-acquired muscle weakness was provided.

- Functional clinical scores may give a prognosis for recovery of walking function of patients with ICU-acquired muscle weakness.

- Limitations are that some of the severely affected ICU patients, for example, patients who were sedated, were excluded from this study.

- Electromyography was not used in all of the included patients for diagnostics of muscle weakness.

whom there is no plausible aetiology other than critical illness. A more precise definition of ICU-acquired muscle weakness includes: (1) weakness must follow the onset of the critical illness; (2) physical examination shows diffuse, symmetric weakness involving all extremities and respiratory muscles; (3) Medical Research Council (MRC) sum score is $<48$ of 60 , or mean MRC score is equal to 4 in all testable muscle groups noted on two occasions separated by $24 \mathrm{~h}$, (4) dependence on mechanical ventilation and (5) causes of weakness not related to the underlying critical illness have been excluded. ${ }^{2}$ The acquired weakness of limb muscles limits significantly activities and assistance for basic activities such as sit to stand or sitting and standing is oftentimes required. ${ }^{3-5}$ This increases morbidity and delays rehabilitation and recovery of walking. ${ }^{6}$ Although full recovery has been reported in approximately $50 \%$ of people with ICU-acquired muscle weakness, improvement is related to the severity of the condition, for example, people with severe 
weakness may take months to improve, or even remain severely affected. ${ }^{8}{ }^{9}$ Focused physical rehabilitation of people with ICU-acquired muscle weakness is therefore of great importance. There is practical evidence that physical rehabilitation of patients can be implemented with few adverse effects. ${ }^{1}{ }^{10}$ In recent years, appropriate assessments were developed and suitable physical intervention strategies were described in the literature. ${ }^{1} 9$ 11-

${ }^{13}$ There are recent longitudinal studies in this field. For instance, Fan et al ${ }^{14}$ investigated 222 survivors of severe critical illness and determined the longitudinal epidemiology of muscle weakness, physical function and health-related quality of life, and their associations with critical illness and ICU exposures. Needham et $a l^{15}$ evaluated muscle strength, a $6 \mathrm{~min}$ walk distance, and the Short Form-36 Physical Function score of 203 survivors after 6 and 12 months of acute lung injury. Semmler et $a l^{16}$ analysed the long-term neuromuscular deficits of survivors of 51 patients with critical illness 6-24 months after discharge from the ICU, measured the MRC sum score, the Overall Disability Sum Score (ODSS), and performed nerve conduction studies and electromyography. MRC sum score and the ODSS score were correlated with the days of ICU treatment and with the days of ventilator support, but the neuromuscular long-term consequences of critical illness were not severe.

Wieske et al investigated post-ICU mortality and physical functioning in 80 patients with acquired weakness at 6 months after ICU discharge. They found that ICUacquired weakness is independently associated with postICU mortality and with lower physical functioning at 6 months after ICU discharge.

Taking all of these essential studies together, one could argue that a detailed knowledge about the exact time course and risk factors of recovery of walking is, however, still not entirely known. From a rehabilitation point of view, it lacks a detailed description of the exact pattern of walking recovery and of physical rehabilitation treatment in the first year of people with ICU-acquired muscle weakness. ${ }^{18}$ Such a depiction could give insights into the particular time course of recovery of walking function of these patients.

Therefore, the aim of the General Weakness Syndrome Therapy (GymNAST) study was to describe and to identify the time course and the pattern of recovery of walking function in these patients. ${ }^{19}$ Another aim of GymNAST was to develop a multivariate risk factor model for recovery of walking function of people with ICU-acquired muscle weakness. We describe the first short-term results of the GymNAST study for walking recovery.

\section{METHODS AND ANALYSIS}

Between January 2013 and March 2015, we screened all patients consecutively from the ICUs of our post-acute ICU and rehabilitation units of the Klinik Bavaria Kreischa in Germany and recruited patients who met our following inclusion and exclusion criteria (as previously reported $^{19}$ ) for our cohort study.

\section{Inclusion criteria}

- Patient is chronically critically ill or has a contemporary history of chronic critical illness. Chronic critical illness was defined as more than 21 days' ICU treatment including mechanical ventilation and at least 14 days' further existing critical situation with the need for ICU treatment) ${ }^{2021}$

- Muscle weakness is defined as a MRC sum score of $<48$ points $^{1}$

- A defined reason for muscle weakness such as a clinical diagnosis of critical illness myopathy (CIM) and polyneuropathy (CIP). The diagnosis of CIM/CIP was performed by a physician in our acute or postacute hospital and always confirmed by a neurologist. Therefore, clinical and (if needed) neurophysiological information was used for diagnosis of CIM/ CIP. The procedure of diagnosis of CIP and CIM is described in detail elsewhere ${ }^{22}$ and will be only briefly described here. All patients underwent a clinical examination by a physician and a specialist in neurology and an electrophysiological workup was performed only by another specialist if the neurologist were in any uncertainty of the clinical diagnosis. ${ }^{22}$ We used this approach because we have recently shown that, in a total of 280 patients with complicated weaning in our post-acute hospital, the positive predictive value of our diagnostic procedure for CIP/ CIM was $97.9 \%$ (95\% CI $69.4 \%$ to $99.9 \%$ ) and the negative predictive value was $88.9 \%(95 \%$ CI $82.7 \%$ to $93.0 \%)^{22}$

- More than or equal to 18 years of age

- Richmond Agitation Sedation Scale (RASS) score from -1 to $2^{23}$

- Written informed consent of the patient or his legal guardian

\section{Exclusion criteria}

- Patients receiving palliative care

- Comorbidities of the trunk or the lower limbs interfering with upright posture and walking function (eg, amputation or fracture of the lower limb)

- Other neuromuscular or neurological diseases and/ or syndromes causing weakness in patients in the ICU (eg, Guillain-Barré syndrome, myasthenia gravis, porphyria, Lambert-Eaton syndrome, amyotrophic lateral sclerosis, vasculitic neuropathy, cervical myelopathy and botulism)

- Severe physical comorbidity before becoming critical ill (eg, frailty due to neurological conditions)

All patients received from the first day of admission to our ICUs of our post-acute ICU and rehabilitation units their individual treatment including physiotherapy and occupational therapy. Physical rehabilitation treatments started, even if patients were mechanically ventilated, on the first day of admission, but differed individually in 
amount and methods due to the severity of critical illness and indication. We did not, however, measure the start, content and amount of treatments in the earlier acute stage.

\section{Measures and outcomes}

We defined walking ability as the primary outcomes of the GymNAST study with more than or equal to 3 of the Functional Ambulation Categories (FAC; ranging from 0 to 5) first described by Holden et al. ${ }^{24}$ FAC is a quick visual measurement of walking, is simple to use and easy to interpret and distinguishes six levels of walking ability on the basis of the amount of physical support required. ${ }^{24}{ }^{25}$ For instance, an FAC of ' 0 ' indicates a patient who is not able to walk at all or needs the help of two therapists (non-functional ambulator) and an FAC of ' 5 ' indicates a patient who can walk everywhere independently, including stairs (independent ambulator). ${ }^{24}{ }^{25}$ Research showed that the FAC has very good reliability, good concurrent and predictive validity, and good responsiveness in neurological rehabilitation. ${ }^{24-26}$ In the present study, we used previously described key questions for every FAC level, used experienced raters and assessed walking ability with FACs. ${ }^{25}$ FACs were measured on a daily basis because we were primarily interested to determine precisely when the good outcome, the ability to walk, occurred in the time course. The definition of a good outcome that we used was a minimum of FAC of ' 3 ' and better (ambulator, dependent on supervision), which indicates a patient who can at least ambulate on level surfaces without manual contact of another person but requires standby guarding of one person either for safety or for verbal cueing. Our primary outcome was therefore analysed as time to event ('event' defined as the time point when ability to walk occurred measured by an FAC of ' 3 ').

\section{Secondary outcomes included}

- Activities of daily living measured with the Barthel Index (BI; 10 items). ${ }^{27}$ The $\mathrm{BI}$ (score range, $0-100$ ) is a valid and reliable index measuring activities of daily life. ${ }^{28}$ Included are 10 items relating to the degree of independence from any help ${ }^{27} 28$

- Clinical severity (eg, mechanical ventilation, dysphagia, tracheostomy) measured with the Early Rehabilitation BI (ERBI) (in the original form described as the Frühreha Index (FRI). ${ }^{29} 30$ The ERBI was designed to allow for a simple determination of clinical severity and contains seven items. Every item will be dichotomously scored as present or absent. These seven items are as translated by Rollnik $2010:^{30}$

- Intensive care supervision ( -50 or 0 points)

- Tracheostomy tube management and supervision ( -50 or 0 points)

- Intermittent (or continuous) mechanical ventilation $(-50$ or 0 points)
- Confused patient (in need of supervision) (-50 or 0)

- Behavioural disturbances (patient being a danger to himself or others) ( -50 or 0 points)

- Severe impairment of communication $(-25$ or 0 points)

- Patient with dysphagia in need of supervision $(-50$ or 0 points)

The sum ERBI score is between 0 and -325 points. Rollnik $^{30}$ described in 2010 high inter-rater reliability for the ERBI $(\mathrm{r}=0.849)$.

- Muscle strength of the upper limbs (shoulder, elbow and wrist) and lower limbs (hip, knee and ankle) using the MRC. We used MRC sum scores for upper and lower limbs ${ }^{131}$

- Grip strength (measured bilaterally using a dynamometer). ${ }^{32} 33$ We summed up the means of both hands. We did not define ICU-acquired weakness on the base of cut-off values from hand grip dynamometry

- Functional Status Score for the ICU (FSS-ICU). ${ }^{34} 35$ The FSS-ICU rates two functional and three additional tasks that are relevant and feasible to perform in the ICU setting. ${ }^{34}{ }^{35}$ All five tasks are evaluated using a seven-point scoring system, with higher scores indicating higher function. ${ }^{34} \mathrm{~A}$ score of 0 will be assigned if a patient is unable to perform a task, either due to physical limitation or medical status 3435

- Physical Function ICU test (scored) (PFIT-s): ${ }^{36} 37$ the PFIT-s is a modified version of the PFIT and contains four items: (1) assistance in sit-to-stand manoeuvres $(0,1$, or 2 people needed), (2) cadence (steps per minute), (3) shoulder flexion strength (muscle strength graded as: $0=$ no contraction, $1=$ visible $/$ palpable muscle contraction, $2=$ movement across gravity, $3=$ movement against gravity, $4=$ movement against gravity with some resistance, or $5=$ movement against gravity with full resistance) and (4) knee extension strength (same muscle strength grading as for shoulder flexion strength) ${ }^{37}$

- Pain using a numeric pain rating scale ${ }^{38}$

- The ability to reach forward as a measure for sit and stance balance. We measured the ability to reach forward while sitting and standing (also called 'functional reach') and summed up the results in $\mathrm{cm}^{39} 40$

- Cognitive measures (Montreal Cognitive Assessment (MoCA $)^{41}$ and clock drawing test (CDT) ${ }^{42} 43$

- Walking speed $(\mathrm{m} / \mathrm{s})$ and walking endurance $(6 \mathrm{~min}$ walking test, 6MWT; metres walked in $6 \mathrm{~min})^{67}$

All assessments and standardised measures were administered by trained and experienced assessors or therapists in the hospital and/or inpatient rehabilitation. We measured patients from baseline (T0) every 2 weeks up to 8 weeks (T4). We defined baseline as the first admission to our post-acute hospital or to our inpatient rehabilitation centre, respectively (T0). On the basis of this definition, the duration of illness was defined as the time from the very first day in the ICU (first admission to the acute 
hospital due to the onset of primary illness) until the study onset ( $\mathrm{T} 0$, baseline, admission to the post-acute hospital or inpatient rehabilitation) or until the observation of the primary outcome or until T1, T2 and so on, respectively. The duration of study was therefore the time from study onset ( $\mathrm{T} 0$, admission to the post-acute hospital or inpatient rehabilitation) until the observation of the primary outcome or T1, T2 and so on, respectively.

We describe here the results of the first 8 weeks of GymNAST as primary or short-term results. We will further describe the results of additional time points and follow-up as long-term results in a separate publication.

\section{Statistical analyses}

We used descriptive analyses, for example, median and IQRs and means and SDs of continuous variables and frequencies and proportions of categorical variables as appropriate. ${ }^{44}$ We applied inference statistics and parametric and non-parametric tests as appropriate. ${ }^{44}$ The global $\alpha$ level was set at 0.05 .

We calculated the probability of regaining walking ability with the method of Kaplan and Meier. ${ }^{45}$ The time to event or censoring was defined as the time between study entry (T0) and the date of reaching an FAC (score 0-5) equal to or more than 3 , or the possible censoring dates of discharge or death, respectively. We used Cox regression analysis to estimate relative hazard rates and to test for differences in variables. ${ }^{46}$ We used univariate and multivariate Cox regression analysis with a selection of possible predictor variables for the primary outcome as follows. ${ }^{46} 47$

\section{Univariate analysis}

These possible predictor variables included: age at study onset, body mass index (BMI), sex, duration of illness, number of medical tubes (catheters and vascular access), duration of mechanical ventilation, number of secondary diagnoses, ERBI item 1, ERBI item 2, ERBI item 3, ERBI item 4, ERBI item 5, ERBI item 6, ERBI item 7, ability to reach forward, FSS-ICU score, PFIT-s, grip strength, MRC sum score upper limb, MRC sum score lower limb, VAS, MoCA and CDT. We did univariate Cox regression analysis of these possible predictor variables and listed the results.

\section{Multivariate analysis and model building}

After the univariate analysis and description of the aforementioned variables, we selected all clinical meaningful and statistical significant variables ( $\alpha$ level of 0.2 for selection) as so-called candidate predictor variables. Afterwards, we used a stepwise regression analysis with all candidate predictor variables. We used for this purpose the procedure proc phreg implemented in SAS/STAT V.9.3; (SAS Institute Inc, Cary, North Carolina, USA). In the process of stepwise regression, a predictor variable had to be significant at the 0.2 level to be entered into the multivariate model and a variable in the model had to be significant at the 0.1 level to remain in the multivariate model. Variables with the highest global score $\chi^{2}$ scores were selected first into a multivariable model. ${ }^{4-50}$ Since the aim of our analysis was to explain the dependent variable (regaining walking function) by a multivariate Cox proportional hazard model with not too many variables (to prevent overfitting), we limited this process to two, three, four and a maximum of five remaining variables in the multivariate model. After that, we compared the multivariate models (with two, three, four and five remaining variables, respectively) on the global score $\chi^{2}$ statistic (so-called best subset selection) and on Akaike's information criterion (AIC) to decide on our final multivariate model. ${ }^{47}$ We expressed the effects of our final multivariate model as HRs with $95 \%$ CIs after a graphical assessment of proportionality of hazards. We used SAS/STAT V.9.3 for all statistical procedures (SAS Institute Inc) and proportional hazards assumptions were tested with the implemented function (proc phreg).

\section{RESULTS}

After screening of 1387 patients between January 2013 and March 2015, we included 150 patients with ICU-acquired muscle weakness ( $30 \%$ female) in our study and analyses (figure 1 and table 1 ).

The demographic and clinical characteristics at each of the individual time points (T0 to T4) can be found in tables 1 and 2.

The primary outcome recovery of walking function was achieved after a median of 28.5 days $(\mathrm{IQR}=45)$ after rehabilitation onset and after a median of 81.5 days $(\mathrm{IQR}=64)$ after onset of illness. The time course of the probability in regaining walking ability is shown in two modes: first, dependent on time from study onset (figure 2A) and second, based on duration of illness (figure 2B). The ability to walk improved significantly over time (as shown graphically in figure 2A,B). The percentage of patients who could walk progressed from T0 $(0 \%)$ to $\mathrm{T} 1(37 \%)$ to $\mathrm{T} 2(68 \%)$ to $\mathrm{T} 3(71 \%)$ to $\mathrm{T} 4$ (85\%; see table 2 for details).

All secondary outcomes except pain improved significantly from T0 to T4 (see table 2). The greatest effects for muscle strength measures (T0 to T4) were found for the MRC sum score upper limbs with a large effect size of 1.28. MRC sum score lower limbs and grip strength, however, improved with an effect size of 0.59 and 0.75 , respectively. The effect sizes for the physical function measures PFIT-s, FSS-ICU, $10 \mathrm{~m}$ walking speed, 6MWT, and functional reach were $0.73,1.19,0.33,1.09$ and 0.99 , respectively. The effect sizes for the cognition measures MOCA and CDT were 0.92 and 0.74, respectively. The effect size for the BI was 1.29.

To explain the dependent variable recovery of walking, a Cox regression analysis was done. The results for every possible predictor variable in our first univariate regression analysis to explain recovery of walking are shown in table 3. After univariate regression analysis, we selected the following candidate predictor variables: age at study onset, BMI, number of medical tubes (catheters and vascular access), duration of mechanical ventilation, 


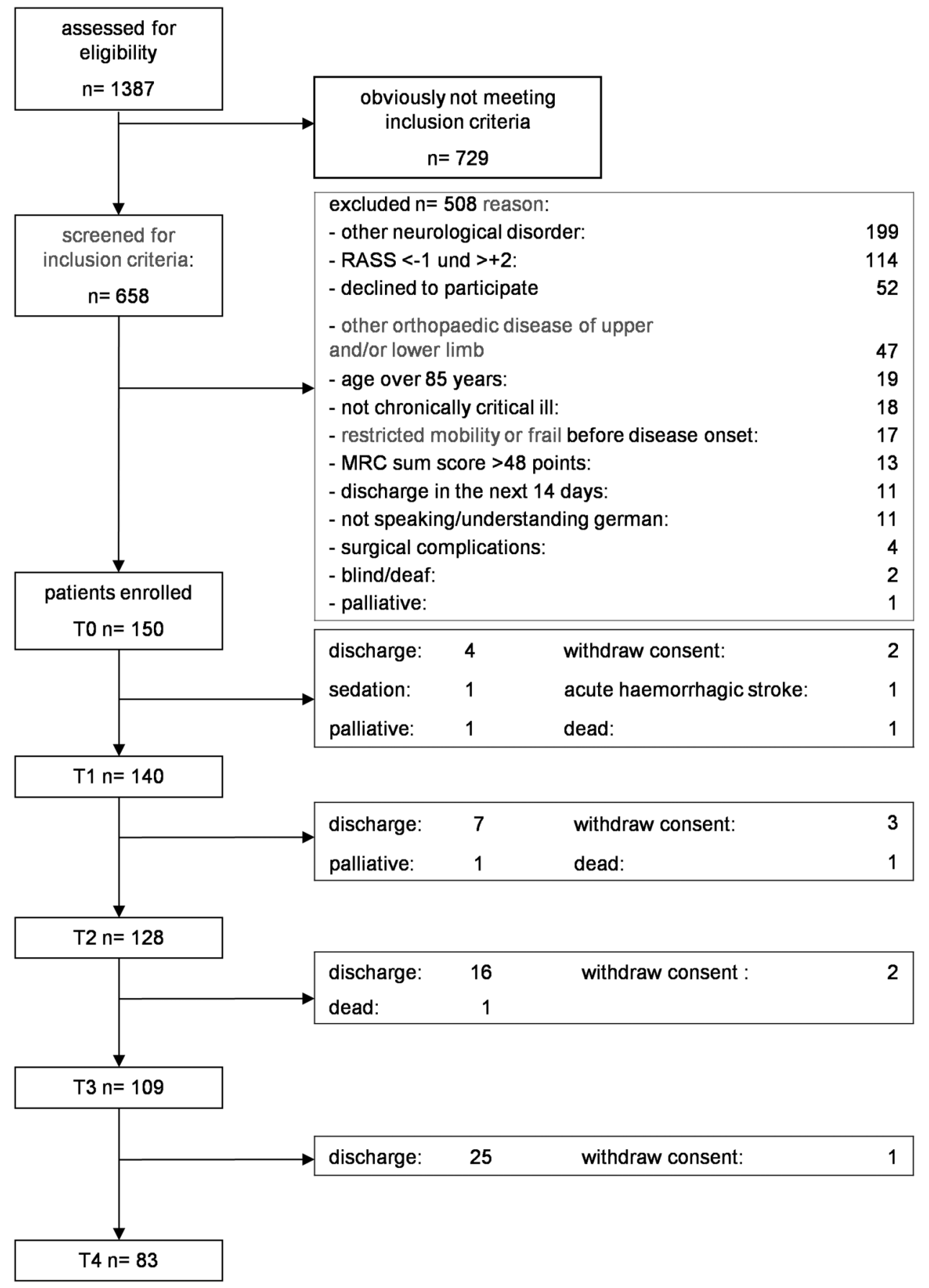

Figure 1 Flow chart (MRC, Medical Research Council; RASS, Richmond Agitation Sedation Scale).

ERBI items 4-7, ability to reach forward, FSS-ICU score, grip strength and MRC sum score upper limb. On the basis of these candidate predictor variables, we did multivariate regression analysis to explain the primary outcome recovery of walking. We compared different multivariate models and selected after statistical and clinical assessment our final multivariate model for recovery of walking. This final model included two variables (model fit statistics $\mathrm{AIC}=656.4$ with covariates): the FSS-ICU score in points (adjusted HR=1.07; 95\% CI 1.03 to 1.12 ) and the ability to reach forward in $\mathrm{cm}$ (adjusted $\mathrm{HR}=1.02 ; 95 \%$ CI 1.00 to 1.04 ; table 4 ).
Physiotherapy was provided between T0 and T1 every week day on average for $45 \mathrm{~min}$. The following main methods of physiotherapy/physical rehabilitation in these $45 \mathrm{~min}$ daily contact time in the first 2 weeks after study onset included: training of sitting balance (in and outside of bed), sit-to-stand training (in and outside of bed), transfer training to get out of bed or to get from bed to wheelchair and vice versa, gait training (including stepping in front of bed), strengthening exercise (in and outside of bed), stepping stairs (including stepping in front of bed) and assistive standing exercises. 
Table 1 Patient characteristics

\begin{tabular}{|c|c|c|}
\hline Variable $(n=150)$ & Median (IQR) & Mean (SD) \\
\hline Age (years) & $71(12)$ & $69.16(9.02)$ \\
\hline BMI (points) & $27.4(6.7)$ & $29.11(8.25)$ \\
\hline Duration of illness (days) & $41(30)$ & $49.13(29.13)$ \\
\hline Duration of mechanical ventilation (days) & $53(42)$ & $65.22(45.14)$ \\
\hline Apache II (points) & $16(5)$ & $16.45(4.08)$ \\
\hline Barthel Index (points) & $5(25)$ & $14.68(19.20)$ \\
\hline MRC sum score at baseline, upper limb & $9.5(3.25)$ & $0.5(0.8)$ \\
\hline MRC sum score at baseline, lower limb & 9 (3.25) & $0.5(0.8)$ \\
\hline MOCA score at baseline (points) & $16(10)$ & $14.3(7.0)$ \\
\hline Primary ICU diagnosis & Frequency (\%) & \\
\hline Sepsis & $82(55)$ & \\
\hline Pneumonia & 29 (19) & \\
\hline Cardiac & $21(14)$ & \\
\hline Other & $18(12)$ & \\
\hline Female & $50(30)$ & \\
\hline Dialysis & $45(30)$ & \\
\hline Patients recruited at post-ICU & $121(81)$ & \\
\hline Patients recruited at inpatient rehabilitation centre & 29 (19) & \\
\hline ERBI item 1: intensive care supervision & $121(81)$ & \\
\hline ERBI item 2: tracheostomy tube management and supervision & $120(80)$ & \\
\hline ERBI item 3: intermittent (or continuous) mechanical ventilation & $103(69)$ & \\
\hline ERBI item 4: confused patient (in need of supervision) & $3(2)$ & \\
\hline ERBI item 5: behavioural disturbances (patient being a danger to himself or others) & $8(5)$ & \\
\hline ERBI item 6: severe impairment of communication & $41(21)$ & \\
\hline ERBI item 7: patient with dysphagia in need of supervision & $81(54)$ & \\
\hline
\end{tabular}

BMI, body mass index; ERBI, Early Rehabilitation Barthel Index; ICU, intensive care unit; MOCA, Montreal Cognitive Assessment; MRC, Medical Research Council.

Table 2 Summary of secondary outcome measures at time points

\begin{tabular}{|c|c|c|c|c|c|c|}
\hline Primary outcome, frequencies & TO & T1 & T2 & T3 & T4 & p Value \\
\hline $\mathrm{FAC}=0$ (in \%) & $105(70)$ & $52(40)$ & $40(35)$ & $26(26)$ & $16(24)$ & $<0.001$ \\
\hline $\mathrm{FAC}=1$ (in \%) & $7(5)$ & $8(6)$ & $3(3)$ & $6(6)$ & $3(4)$ & \\
\hline $\mathrm{FAC}=2($ in $\%)$ & $38(25)$ & $3(2)$ & $5(4)$ & $12(12)$ & $3(4)$ & \\
\hline $\mathrm{FAC}=3$ (in \%) & $0(0)$ & $30(23)$ & $22(19)$ & $13(13)$ & $15(22)$ & \\
\hline $\mathrm{FAC}=4$ (in \%) & $0(0)$ & $30(23)$ & $29(25)$ & $28(28)$ & $21(31)$ & \\
\hline $\mathrm{FAC}=5$ (in \%) & $0(0)$ & $7(5)$ & $16(14)$ & $16(16)$ & $9(13)$ & \\
\hline \multicolumn{7}{|l|}{ Muscle strength measures } \\
\hline MRC sum score upper limbs* & $9.50(2.55)$ & $11.50(2.46)$ & $12.00(2.49)$ & $12.00(2.27)$ & $12.50(2.15)$ & $<0.001$ \\
\hline MRC sum score lower limbs* & $9.00(3.25)$ & $10.50(3.00)$ & $10.50(3.00)$ & $11.00(3.50)$ & $11.00(3.50)$ & $<0.001$ \\
\hline Grip strength (in kg)† & $9.33(5.35)$ & $11.92(6.22)$ & $13.32(6.99)$ & $13.54(6.18)$ & $14.19(7.66)$ & $<0.001$ \\
\hline \multicolumn{7}{|l|}{ Physical function measures } \\
\hline PFIT-s (points) ${ }^{\star}$ & $4.00(6.00)$ & $8.00(5.50)$ & $8.00(5.00)$ & $8.00(6.00)$ & $8.00(5.00)$ & $<0.001$ \\
\hline FSS-ICU score (points) ${ }^{\star}$ & $16.0(15.0)$ & $25.0(16.0)$ & $30.0(14.0)$ & $29.0(13.0)$ & $31.5(11.0)$ & $<0.001$ \\
\hline $10 \mathrm{~m}$ walking speed $(\mathrm{m} / \mathrm{s}) \dagger$ & $0.24(0.25)$ & $0.50(0.50)$ & $0.51(0.53)$ & $0.45(0.48)$ & $0.35(0.42)$ & $<0.001$ \\
\hline $6 \mathrm{MWT}(\mathrm{m}) \dagger$ & $25.8 \pm 60.0$ & $87.1 \pm 109.7$ & $114.2 \pm 126.3$ & $112.8 \pm 121.0$ & $126.3 \pm 125.1$ & $<0.001$ \\
\hline Pain (mm VAS)† & $4.0 \pm 8.3$ & $7.6 \pm 12.3$ & $6.2 \pm 10.7$ & $6.2 \pm 9.8$ & $4.6 \pm 8.3$ & 0.751 \\
\hline Functional reach $(\mathrm{cm}) \dagger$ & $31.9 \pm 23.4$ & $46.9 \pm 23.5$ & $50.6 \pm 25.9$ & $49.7 \pm 24.8$ & $54.4 \pm 22.2$ & $<0.001$ \\
\hline \multicolumn{7}{|l|}{ Cognition measures } \\
\hline MOCA (points)† & $14.3 \pm 7.0$ & $17.1 \pm 7.4$ & $18.9 \pm 6.6$ & $19.8 \pm 6.3$ & $20.4 \pm 6.3$ & $<0.001$ \\
\hline CDT (points) $\dagger$ & $3.9 \pm 1.8$ & $3.2 \pm 1.6$ & $2.9 \pm 1.4$ & $2.6 \pm 1.6$ & $2.6 \pm 1.7$ & $<0.001$ \\
\hline \multicolumn{7}{|l|}{ Activities and mobility } \\
\hline $\mathrm{BI}$ (points) $)^{\star}$ & $5.0(25.0)$ & $35.0(55.0)$ & $45.0(65.0)$ & $50.0(60.0)$ & $60.0(60.0)$ & $<0.001$ \\
\hline
\end{tabular}

*Presented as median and IQRs.

†Presented as means and SDs.

6MWT, 6 min walking test; BI, Barthel Index; CDT, clock drawing test; FAC, Functional Ambulation Categories; FSS-ICU, Functional Status Score for the intensive care unit scored; MOCA, Montreal Cognitive Assessment; MRC, Medical Research Council (muscle strength of the upper limbs (sum of shoulder, elbow and wrist) and lower limbs (sum of hip, knee and ankle)); PFIT-s, Physical Function ICU test scored; T, time point; VAS, visual analogue scale. 

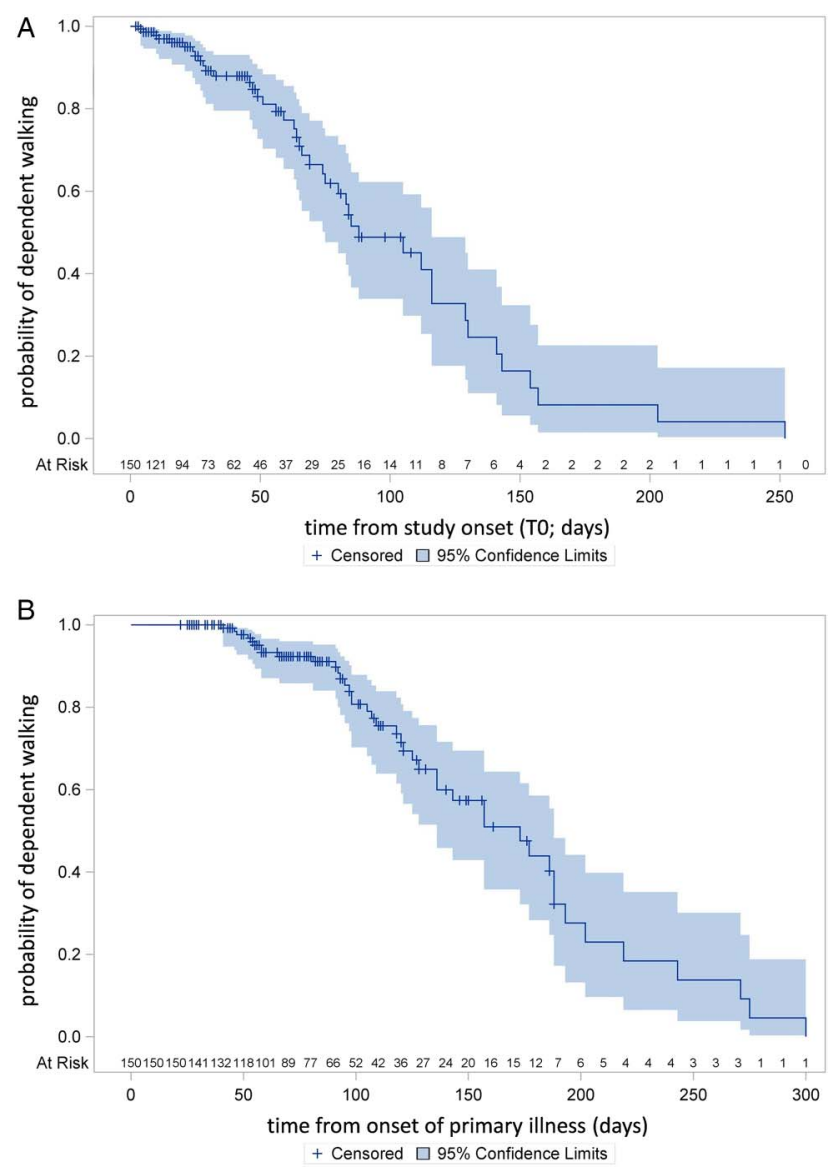

Figure 2 (A) Time course of recovery of walking function from study onset (TO). (B) Time course of recovery of walking function from onset of the primary illness.

\section{DISCUSSION}

This study is one of the first studies with rigorous repeated measures design over the time course of 1 year of people with ICU-acquired muscle weakness.

As a main result, we found that $50 \%$ of all included patients were able to walk at a median of 28.5 days after rehabilitation and after a median duration of illness of 81.5 days.

We used a wide range of functional variables to describe the pattern of regaining walking. The main variables in our final multivariate model to explain the ability to walk, however, were clinical scales, the FSS-ICU score and the ability to reach forward in sitting and standing at baseline. Both assessments can be used very early and very easily in patients in the ICU and may predict the recovery of walking ability of people with ICU-acquired muscle weakness.

To the best of our knowledge, many prognostic studies including people with ICU-acquired muscle weakness used a rather conventional prognostic design using a baseline test and compared with ICU discharge and follow-up data ${ }^{6} 3637$ and just some studies measured functional recovery continuously over time. ${ }^{51}$ Instead of comparing two or more measurements of the patient's performance, however, it seems to be more informative to analyse the dynamic recovery systematically using equal time intervals over an appropriate time period, for example, with daily assessments of walking ability. Our study might therefore provide a more detailed understanding of a pattern and the dynamics of recovery of walking ability of chronically ill people with ICU-acquired muscle weakness.

As recently shown, there are no randomised trials so far including people with ICU-acquired muscle weakness with a diagnosis of CIP or CIM. ${ }^{18}$ To the best of our knowledge, cohort studies describing the detailed recovery pattern of walking ability in people with ICU-acquired muscle weakness are also quite rare.

One recent study of Denehy et al ${ }^{12}$ included 150 people after 5 or more days of ICU admission but did not use a defined diagnosis of CIP or CIM as inclusion criteria. Compared to the population of Denehy et $a l^{12}$ our patients had a longer length of hospital stay in the acute hospital (median 41 days vs 20-23.5 days) and a longer duration of mechanical ventilation (median 53 days vs $98 \mathrm{~h}$ ) and only $17-21 \%$ of patients included in Denehy's trial had ICU-acquired muscle weakness.

Compared to Fan et $a l^{14}$ who described a mean Apache II of 26 points, our study population, however, had a mean of 16 points and could therefore be seen to be somewhat less severe affected. Given the long duration of illness, however, our patients were more chronically affected.

A recent multicentre cohort study investigated functional recovery at 6 months among 192 mechanically ventilated ICU patients (about 50\% of these patients had ICU-acquired weakness).$^{52}$ The authors, however, did not describe the detailed functional recovery of walking in this population.

Cuthbertson et a $\tilde{\rho}^{3}$ investigated 286 patients after discharge from intensive care and 192 patients completed the 1 year follow-up, but a defined diagnosis to CIP or CIM as a cause for muscle weakness was not used as an inclusion criterion. The authors conclude, however, that further work should focus on the recovery from critical illness. ${ }^{53}$

In our study, we chose the PFIT-s and FSS-ICU as main clinical assessments. Both measures are common and recommended for patients in the ICU and were well described in this population. ${ }^{34-37}$ Other studies used the Rivermead Mobility Index, a scale well known in stroke rehabilitation. ${ }^{54}$ Nordon-Craft $e t a l^{36}$ described on the basis of 51 patients from the ICU that the PFIT-s was highly correlated to MRC sum score and grip strength. Additionally, at ICU discharge, an MRC sum score cut point of 41.5 predicted a subject's ability to perform the standing components of the PFIT-s. ${ }^{36}$ In our study, however, we could not find predictive values for the MRC sum score or for the PFIT-s. We found the best prediction for walking recovery from a model containing the FSS-ICU score and the functional reach in the first week of rehabilitation. 
Table 3 Summary of the univariate Cox proportional hazards for regaining walking ability of all potential predictor variables

\begin{tabular}{|c|c|c|c|c|}
\hline Variable (at T0) & $\chi^{2}$ & p Value & HR & $95 \% \mathrm{Cl}$ \\
\hline Age (years) & 7.37 & 0.007 & 0.970 & 0.949 to 0.992 \\
\hline $\mathrm{BMI}$ & 3.92 & 0.048 & 0.972 & 0.944 to 1.000 \\
\hline Sex (male) & 0.00 & 0.996 & 1.001 & 0.637 to 1.573 \\
\hline Duration of illness (days) & 1.33 & 0.249 & 0.995 & 0.986 to 1.004 \\
\hline Number of medical tubes (catheters and vascular access) & 1.83 & 0.176 & 0.901 & 0.774 to 1.048 \\
\hline Duration of mechanical ventilation (days) & 8.05 & 0.005 & 0.992 & 0.986 to 0.997 \\
\hline Number of secondary diagnoses & 0.07 & 0.790 & 0.996 & 0.965 to 1.03 \\
\hline ERBI item 1: intensive care supervision & 1.37 & 0.242 & 1.009 & 0.994 to 1.023 \\
\hline ERBI item 2: tracheostomy tube management and supervision & 0.41 & 0.524 & 1.005 & 0.990 to 1.019 \\
\hline ERBI item 3: intermittent or continuous mechanical ventilation & 0.00 & 0.986 & 1.000 & 0.987 to 1.014 \\
\hline ERBI item 4: confused patient (in need of supervision) & 2.14 & 0.144 & 1.023 & 0.992 to 1.055 \\
\hline $\begin{array}{l}\text { ERBI item 5: behavioural disturbances } \\
\text { (patient being a danger to himself or others) }\end{array}$ & 2.37 & 0.124 & 0.984 & 0.965 to 1.004 \\
\hline ERBI item 6: severe impairment of communication & 11.24 & 0.001 & 1.037 & 1.015 to 1.060 \\
\hline ERBI item 7: patient with dysphagia in need of supervision & 2.43 & 0.119 & 0.993 & 0.983 to 1.002 \\
\hline Ability to reach forward $(\mathrm{cm})$ & 4.06 & 0.044 & 1.028 & 1.001 to 1.056 \\
\hline FSS-ICU score (points) & 1.99 & 0.159 & 1.062 & 0.977 to 1.115 \\
\hline PFIT-s (points) & 0.51 & 0.475 & 1.095 & 0.854 to 1.403 \\
\hline Grip strength $(\mathrm{kg})$ & 3.03 & 0.082 & 1.075 & 0.991 to 1.167 \\
\hline MRC sum score upper limb (points) & 8.44 & 0.004 & 0.715 & 0.571 to 0.897 \\
\hline MRC sum score lower limb (points) & 0.00 & 0.970 & 1.004 & 0.808 to 1.248 \\
\hline VAS (mm) & 0.43 & 0.514 & 1.012 & 0.977 to 1.047 \\
\hline MoCA (points) & 1.34 & 0.247 & 0.960 & 0.896 to 1.029 \\
\hline CDT (points) & 1.23 & 0.267 & 0.847 & 0.632 to 1.136 \\
\hline
\end{tabular}

BMI, body mass index; CDT, clock drawing test; ERBI, Early Rehabilitation Barthel Index; FSS-ICU, Functional Status Score for the intensive care unit; MoCA, Montreal Cognitive Assessment; MRC, Medical Research Council; PFIT-s, Physical Function ICU test (scored); VAS, visual analogue scale.

On the one hand, this study shows at first glance good recovery of walking function measured with the FAC. On the other hand, comparing with reference values of healthy persons from six countries for the 6MWTs and the walking speed, we found that many of our patients were still reasonably below the 10th centile of age-adjusted walking distance and speed. ${ }^{55}$ This shows clearly that the recovery of walking even after 8 weeks of physical rehabilitation is still not at a normal level. Further studies should therefore provide insights into specific treatment approaches to improve the walking speed and distance in patients with ICU-acquired muscle weakness. $^{18}{ }^{56}$ In practice, a long-term treatment approach seems warranted for this chronically ill population.

At first look, it seems a bit strange that the average walking speed improved from $\mathrm{T} 0$ to $\mathrm{T} 3$ and then declined to T4. This is, however, due to the fact that patients with good recovered walking function were discharged earlier (and therefore excluded from the analysis) compared to patients with not well-recovered walking function.

Eventually, the patients included in our study were relatively chronically and severely ill, had all ICU-acquired muscle weakness and were therefore not directly comparable to other published clinical trials in the field of ICU research.

Strong aspects of GymNAST are the prospective design and multiple repeated assessments during the first months of illness using equal time intervals of people with ICU-acquired muscle weakness with daily assessment of walking ability. This study might therefore provide new and more detailed information about the short-term pattern of walking recovery and the physical rehabilitation content of people with ICU-acquired muscle weakness.

A potential limitation of the study is that the very seriously affected patients in terms of those who were very

Table 4 Summary of the final multivariate Cox proportional hazard model for regaining walking ability

\begin{tabular}{lllll}
\hline Variable & $\chi^{2}$ & p Value & HR & $\mathbf{9 5 \% ~ C l ~}$ \\
\hline FSS-ICU score in points & 13.36 & 0.0003 & 1.074 & 1.033 to 1.115 \\
Ability to reach forward in cm & 5.25 & 0.0219 & 1.019 & 1.003 to 1.036
\end{tabular}

Higher scores of the FSS-ICU and the greater ability to reach forward at T0 are indicating significantly higher chances to regain walking ability.

FSS-ICU score, Functional Status Score intensive care unit. 
sedated or very agitated, who were not able to perform the assessments, were not included, thereby reducing the possibility to generalise the results to the whole critical ill population. Diagnosis of CIP and CIM requires clinical evaluation and electrophysiological investigations. ${ }^{57}$ Therefore another limitation is that an electrophysiological evaluation was not always provided. The limitations of this study are that electromyography was not used for differential diagnostics of muscle weakness, for example, between CIM and CIP and for other reasons of acquired muscle weakness; and that creatine kinase was not measured.

Further studies should use a randomised controlled design, should include people with ICU-acquired muscle weakness with a defined reason for muscle weakness such as a defined diagnosis of CIP and/or CIM and should investigate specific rehabilitation therapies to improve or to speed up walking recovery in this population with ICU-acquired muscle weakness.

Contributors JM, SM, FO and MP planned the study. FO, JM and MP contributed to the procurement of funding. JM., SM and MP developed the protocol, SM, MP and JM evaluated and interpreted the data, JM, SM and MP did the statistical analysis. All the authors contributed to the writing and checked the final draft of the manuscript.

Funding This study is financially supported by the Centre of Research from Klinik Bavaria, Kreischa, Germany and by the Department of Public Health, Medizinische Fakultät 'Carl Gustav Carus', Technische Universität Dresden, Germany.

\section{Competing interests None declared.}

Patient consent Obtained.

Ethics approval We conducted this study in accordance with the 'Helsinki Declaration' and received ethical approval by the local ethic commission (Sächsische Landesärztekammer, EK-BR-32/13-1/106755) and registered the study before publication (German Register of Clinical Trials, DRKS00006528).

Provenance and peer review Not commissioned; externally peer reviewed.

Data sharing statement No additional data are available.

Open Access This is an Open Access article distributed in accordance with the Creative Commons Attribution Non Commercial (CC BY-NC 4.0) license, which permits others to distribute, remix, adapt, build upon this work noncommercially, and license their derivative works on different terms, provided the original work is properly cited and the use is non-commercial. See: http:// creativecommons.org/licenses/by-nc/4.0/

\section{REFERENCES}

1. Nordon-Craft A, Moss M, Quan D, et al. Intensive care unit-acquired weakness. Phys Ther 2012;92:1494-506.

2. Stevens RD, Marshall SA, Cornblath DR, et al. A framework for diagnosing and classifying intensive care unit-acquired weakness. Crit Care Med 2009;37(10 Suppl):S299-308.

3. Fan E. Critical illness neuromyopathy and the role of physical therapy and rehabilitation in critically ill patients. Respir Care 2012;57:933-44; discussion 944-6.

4. Herridge MS. The challenge of designing a post-critical illness rehabilitation intervention. Crit Care 2011;15:1002.

5. Ohtake PJ, Strasser DC, Needham DM. Rehabilitation for people with critical illness: taking the next steps. Phys Ther 2012;92:1484-8.

6. Herridge MS, Cheung AM, Tansey CM, et al. One-year outcomes in survivors of the acute respiratory distress syndrome. $N$ Engl J Med 2003;348:683-93.
7. Herridge MS, Tansey CM, Matté A, et al. Functional disability 5 years after acute respiratory distress syndrome. $N$ Engl J Med 2011;364:1293-304.

8. Hermans $\mathrm{G}$, De Jonghe $\mathrm{B}$, Bruyninckx $\mathrm{F}$, et al. Clinical review: critical illness polyneuropathy and myopathy. Crit Care 2008;12:238.

9. Kress J, Hall J. ICU-acquired weakness and recovery from critical illness. N Engl J Med 2014;370:1626-35.

10. Fan E, Cheek F, Chlan L, et al. An official American Thoracic Society Clinical Practice guideline: the diagnosis of intensive care unit-acquired weakness in adults. Am J Respir Crit Care Med 2014;190:1437-46.

11. Denehy L, Berney S, Skinner E, et al. Evaluation of exercise rehabilitation for survivors of intensive care: protocol for single blind randomised controlled trial. Open Crit Care Med J, 2008;00:39-47.

12. Denehy L, Skinner EH, Edbrooke L, et al. Exercise rehabilitation for patients with critical illness: a randomized controlled trial with 12 months follow up. Crit Care 2013;17:R156.

13. Nordon-Craft A, Schenkman M, Ridgeway K, et al. Physical therapy management and patient outcomes following ICU-acquired weakness: a case series. J Neurol Phys Ther 2011;35:133-40.

14. Fan E, Dowdy DW, Colantuoni E, et al. Physical complications in acute lung injury survivors: a two-year longitudinal prospective study. Crit Care Med 2014;42:849-59.

15. Needham DM, Wozniak AW, Hough CL, et al. Risk factors for physical impairment after acute lung injury in a national, multicenter study. Am J Respir Crit Care Med 2014;189:1214-24.

16. Semmler A, Okulla $\mathrm{T}$, Kaiser $\mathrm{M}$, et al. Long-term neuromuscular sequelae of critical illness. J Neurol 2013;260:151-7.

17. Wieske L, Dettling-Ihnenfeldt DS, Verhamme C, et al. Impact of ICU-acquired weakness on post-ICU physical functioning: a follow-up study. Crit Care 2015;19:196.

18. Mehrholz J, Pohl M, Burridge J, et al. Physical rehabilitation for critical illness myopathy and neuropathy. Cochrane Database Syst Rev 2015;3:CD010942.

19. Mehrholz J, Mückel S, Oehmichen F, et al. The General Weakness Syndrome Therapy (GymNAST) study: protocol for a cohort study on recovery on walking function. BMJ Open 2014;4:e006168.

20. Oehmichen F, Ragaller M. Beatmungsentwöhnung bei Chronisch-Kritisch-Kranken. Intensiv- und Notfallbehandlung 2012;37:118-26.

21. Nelson JE, Cox CE, Hope AA, et al. Chronic critical illness. Am J Respir Crit Care Med 2010;182:446-54.

22. Oehmichen F, Pohl M, Schlosser R, et al. Critical-illness-Polyneuropathie und -Polymyopathie. Wie sicher ist die klinische Diagnose bei Patienten mit Weaning-Versagen? [Critical illness polyneuropathy und polymyopathy. How certain is the clinical diagnosis in patients with weaning failure?]. Nervenarzt 2012;83:220-5

23. Sessler CN, Gosnell MS, Grap MJ, et al. The Richmond Agitation-Sedation Scale: validity and reliability in adult intensive care unit patients. Am J Respir Crit Care Med 2002;166:1338-44.

24. Holden MK, Gill KM, Magliozzi MR, et al. Clinical gait assessment in the neurologically impaired. Reliability and meaningfulness. Phys Ther 1984;64:35-40.

25. Mehrholz J, Wagner K, Rutte K, et al. Predictive validity and responsiveness of the Functional Ambulation Category in hemiparetic patients after stroke. Arch Phys Med Rehabil 2007;88:1314-19.

26. Holden MK, Gill KM, Magliozzi MR. Gait assessment for neurologically impaired patients. Standards for outcome assessment. Phys Ther 1986;66:1530-9.

27. Mahoney FI, Barthel DW. Functional Evaluation: The Barthel Index. Md State Med J 1965;14:61-5.

28. Wade DT, Collin C. The Barthel ADL Index: a standard measure of physical disability? Int Disabil Stud 1988;10:64-7.

29. Pohl M, Bertram M, Hoffmann B, et al. Der Frühreha-Index: Ein Manual zur Operationalisierung. Rehabilitation 2010;49:22-9.

30. Rollnik J. The Early Rehabilitation Barthel Index (ERBI). Rehabilitation (Stuttg) 2011;50:408-11

31. Kleyweg RP, van der Meché FG, Schmitz PI. Interobserver agreement in the assessment of muscle strength and functional abilities in Guillain-Barre syndrome. Muscle Nerve 1991;14:1103-9.

32. Mathiowetz V, Weber K, Volland G, et al. Reliability and validity of grip and pinch strength evaluations. J Hand Surg Am 1984; 9:222-6.

33. Mathiowetz V, Kashman N, Volland G, et al. Grip and pinch strength normative data for adults. Arch Phys Med Rehabil 1985;66:69-74.

34. Zanni JM, Korupolu R, Fan E, et al. Rehabilitation therapy and outcomes in acute respiratory failure: an observational pilot project. $J$ Crit Care 2010;25:254-62.

35. Thrush A, Rozek M, Dekerlegand J. The clinical utility of the functional status score for the intensive care unit (FSS-ICU) at a 
long-term acute care hospital: a prospective cohort study. Phys Ther 2012;92:1536-45.

36. Nordon-Craft A, Schenkman M, Edbrooke L, et al. The physical function intensive care test: implementation in survivors of critical illness. Phys Ther 2014;94:1499-507.

37. Denehy L, de Morton N, Skinner E, et al. A physical function test for use in the intensive care unit: validity, responsiveness, and predictive utility of the physical function ICU test (scored). Phys Ther 2013;93:1636-45.

38. Yu A, Teitelbaum J, Scott J, et al. Evaluating pain, sedation, and delirium in the neurologically critically ill-feasibility and reliability of standardized tools: a multi-institutional study. Crit Care Med 2013;41:2002-7.

39. Weiner DK, Duncan PW, Chandler J, et al. Functional reach: a marker of physical frailty. J Am Geriatr Soc 1992;40:203-7.

40. Newton RA. Validity of the multi-directional reach test: a practical measure for limits of stability in older adults. $J$ Gerontol A Biol Sci Med Sci 2001;56:M248-52.

41. Nasreddine ZS, Phillips NA, Bédirian V, et al. The Montreal Cognitive Assessment, MoCA: a brief screening tool for mild cognitive impairment. J Am Geriatr Soc 2005;53:695-9.

42. Ismail Z, Rajji TK, Shulman KI. Brief cognitive screening instruments: an update. Int J Geriatr Psychiatry 2010;25:111-20.

43. Shulman KI. Clock-drawing: is it the ideal cognitive screening test? Int J Geriatr Psychiatry 2000;15:548-61.

44. Armitage P, Colton T. Encyclopedia of biostatistics. Chichester: Wiley, 1998.

45. Kaplan E, Meier P. Nonparametric estimation from incomplete observations. J Am Stat Assoc 1958;53:457-81.

46. Kleinbaum D, Klein M. Survival analysis. A self-learning text. 3rd edn. New York: Springer, 2012.

47. Hosmer D, Lemeshow S, May S. Applied survival analysis: regression modeling of time to event data. 2nd edn. New York: John Wiley \& Sons, Inc, 2008.
48. Spiegelhalter DJ. Probabilistic prediction in patient management and clinical trials. Stat Med 1986;5:421-33.

49. Steyerberg EW, Eijkemans MJ, Harrell FE Jr, et al. Prognostic modelling with logistic regression analysis: a comparison of selection and estimation methods in small data sets. Stat Med 2000;19:1059-79.

50. Hosmer DW, Lemeshow S. Applied logistic regression. 2ed edn. New York: John Wiley \& Sons, Inc., 2000.

51. Schweickert WD, Pohlman MC, Pohlman AS, et al. Early physical and occupational therapy in mechanically ventilated, critically ill patients: a randomised controlled trial. Lancet, 2009;373:1874-82.

52. Hodgson C, Bellomo R, Berney S, et al. Early mobilization and recovery in mechanically ventilated patients in the ICU: a bi-national, multi-centre, prospective cohort study. Crit Care 2015;19:81.

53. Cuthbertson BH, Rattray J, Campbell MK, et al. The PRaCTICaL study of nurse led, intensive care follow-up programmes for improving long term outcomes from critical illness: a pragmatic randomised controlled trial. BMJ 2009;339:b3723.

54. Walsh TS, Salisbury LG, Merriweather JL, et al, RECOVER Investigators. Increased Hospital-Based Physical Rehabilitation and Information Provision After Intensive Care Unit Discharge: The RECOVER Randomized Clinical Trial. JAMA Intern Med 2015;175:901-10.

55. Casanova C, Celli BR, Barria P, et al. The 6-min walk distance in healthy subjects: reference standards from seven countries. Eur Respir J 2011;37:150-6.

56. Connolly B, Salisbury L, O'Neill B, et al. Exercise rehabilitation following intensive care unit discharge for recovery from critical illness. Cochrane Database Syst Rev 2015;6:CD008632.

57. Latronico N, Bolton C. Critical illness polyneuropathy and myopathy: a major cause of muscle weakness and paralysis. Lancet Neurolology 2011;10:931-41. 